\title{
First Report on a Semicrystalline Vinyl Polyperoxide
}

\author{
J. JAYASEHARAN, ${ }^{1 *}$ K. KISHORE, ${ }^{1}$ G. NALINI, ${ }^{2}$ T. N. GURUROW ${ }^{2}$ \\ ${ }^{1}$ Department of Inorganic and Physical Chemistry, Indian Institute of Science, Bangalore 560012, India \\ ${ }^{2}$ Department of Solid State and Structural Chemistry Unit, Indian Institute of Science, Bangalore 560012, India
}

Received 3 May 1999; accepted 30 July 1999

\begin{abstract}
Vinyl polyperoxides constitute a narrow but important class of polymers because they show several novel physicochemical properties. Having a highly flexible backbone, they are also highly viscous amorphous materials. Interestingly, we found that $\operatorname{poly}(\alpha$-phenyl styrene peroxide) (PAPSP) forms a crystalline structure that is perhaps the first crystalline structure known in the family of polyperoxides. From detailed powder X-ray diffraction analysis and simulation studies, PAPSP was found to have a helical conformation. Another interesting feature of PAPSP is that it is highly thermally stable and photostable compared to other known polyperoxides. This makes PAPSP the most eligible candidate for exploring novel application potentials. () 1999 John Wiley \& Sons, Inc. J Polym Sci A: Polym Chem 37: 4033-4036, 1999
\end{abstract}

Keywords: $\operatorname{poly}(\alpha$-phenyl styrene peroxide); vinyl polyperoxide; crystalline structure; simulation; helical structure; dihedral angle

\section{INTRODUCTION}

Regularity in structure, strong intermolecular forces, and sufficient flexibility are some of the necessary requirements for polymer chains to form a crystalline order. Highly flexible or highly rigid polymer chains do not induce crystallinity. Symmetry and regularity can be introduced into a polymer chain through tacticity. Isotactic and syndiotactic polymers readily form crystalline structures. Solely on the basis of symmetry and regularity, linear polyethylene crystallizes to a very high degree even in the absence of tacticity and strong intermolecular forces. Polyperoxides, being highly flexible, generally do not form crystalline structures. In fact, no crystallinity in

This paper is dedicated in memory of Professor Kaushal Kishore, who passed away on the 2nd March, 1999.

* Present address: Department of Biomedical Engineering, 512 Wickenden Building, Case Western Reserve University, Cleveland, $\mathrm{OH} 44106$

Correspondence to: J. Jayaseharan (E-mail: jxj40@po. cwru.edu)

Journal of Polymer Science: Part A: Polymer Chemistry, Vol. 37, 4033-4036 (1999) (c) 1999 John Wiley \& Sons, Inc. polyperoxides is known, although more than a dozen of them have been reported so far. ${ }^{1-8}$ In an effort to obtain a crystalline polyperoxide that has a simple and experimentally realizable structure, we found $\operatorname{poly}(\alpha$-phenyl styrene peroxide) (PAPSP) to be a suitable candidate. It has the required symmetry and sufficient flexibility, although tacticity and intermolecular forces are absent just as in polyethylene. Indeed, to our utmost satisfaction, this polyperoxide does form a crystalline structure as shown by X-ray diffraction (XRD). This is the first report of a crystalline polyperoxide. Interestingly, PAPSP was the first polyperoxide reported by Staudinger, ${ }^{9}$ but its spectral characterizations are not available so far. Except for the kinetics of its polymerization by Mayo et al., ${ }^{10}$ no other studies on PAPSP have been reported.

\section{EXPERIMENTAL}

PAPSP was purified by repeated precipitation from chloroform solution and dried under vac- 


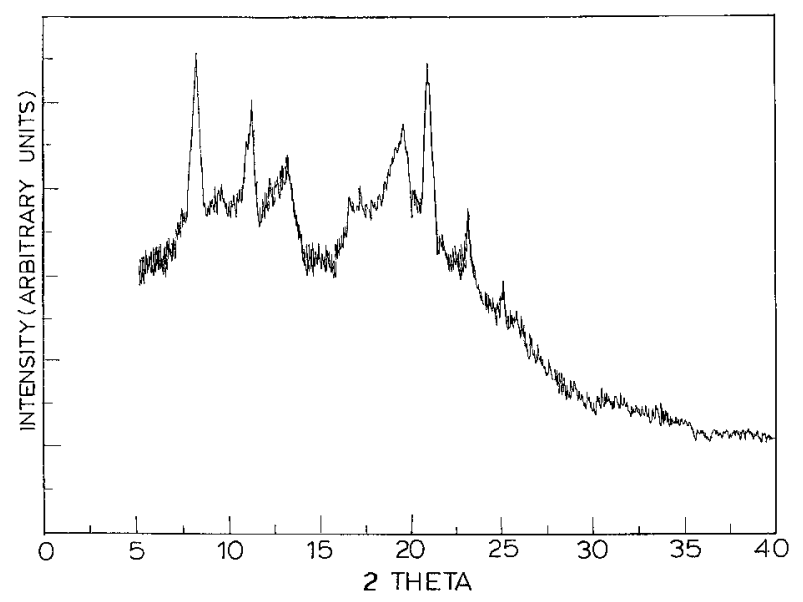

Figure 1. Experimentally obtained powder XRD pattern of PAPSP.

uum. The number, viscosity, weight, $Z$ average molecular weight, and polydispersity were found to be $2388,4458,5572,12,392$, and 2.33 , respectively. ${ }^{11}$ PAPSP was characterized spectroscopically, ${ }^{12}$ and the results supported the alternating structure of a vinyl monomer and oxygen generally observed in vinyl polyperoxides. Models were built using the Biosym-Insight II molecular modeling program (Molecular Simulations Inc., San Diego, CA). XRD simulations were performed with the Discover 95.0 subroutine in the solid builder mode using the module characterize. The Powder XRD patterns of PAPSP were obtained on a STOE STADI/ $\mathrm{P}$ machine in the transmission mode $\left(2 \theta=5-40^{\circ}\right)$ with a precalibrated curved position-sensitive detector. The XRD patterns were taken with monochromatized $\mathrm{Cu}-\mathrm{K}_{\alpha}(\lambda$ $=1.54056 \AA$ ) radiation. Finely ground powder samples were mounted on the transmission geometry holder, and the samples were rotated during data collection to avoid any orientational effects.

\section{RESULTS AND DISCUSSION}

\section{Experimental XRD Pattern}

The XRD pattern (Fig. 1) shows significant crystallinity as well as an amorphous halo that suggests that PAPSP is a semicrystalline polymer. From the area under the diffraction peaks and amorphous halo, the crystallinity in PAPSP was found to be $\sim 60 \%$. Because of the significant amorphous content, it was not possible to obtain a single crystal of PAPSP, so powder XRD was the only option for obtaining any crystal-structure information. To analyze the crystalline structure, we, using a software procedure developed locally, recast the XRD pattern presented in Figure 1 to display a diffractogram devoid of amorphous halo features (Fig. 2), and the amorphous background was subtracted with a third-order polynomial. The powder XRD pattern of PAPSP was indexed as a monoclinic system with the following cell dimensions: $\mathrm{a}=11.83 \AA, \mathrm{b}=10.71 \AA, \mathrm{c}=8.55 \AA$, $\beta=97.97^{\circ}, \mathrm{V}=1073.5 \AA{ }^{3}$ and figure of merit $=15.5$ (with the program TREOR). ${ }^{13}$ The crystalline domain size, which was estimated using the Scherrer formula, ${ }^{14}$ ranged from 49 to $200 \AA$. Table I lists the $d$ spacings, domain size $(L), 2 \theta$ values, relative intensity, and possible indexing in the monoclinic symmetry. Because the number of observed reflections in the XRD pattern were very limited, the determination of the complete structure of the polymer was not viable. However, the basic features of the polymeric structure, in terms of repeat subunits, could be modeled and cross-checked for relative comparisons with the observed XRD patterns (Table I). ${ }^{15}$ Still, it was difficult to examine the model in depth using the Rietveld refinement ${ }^{16}$ procedure because of the large extent $(\sim 40 \%)$ of the amorphous halo.

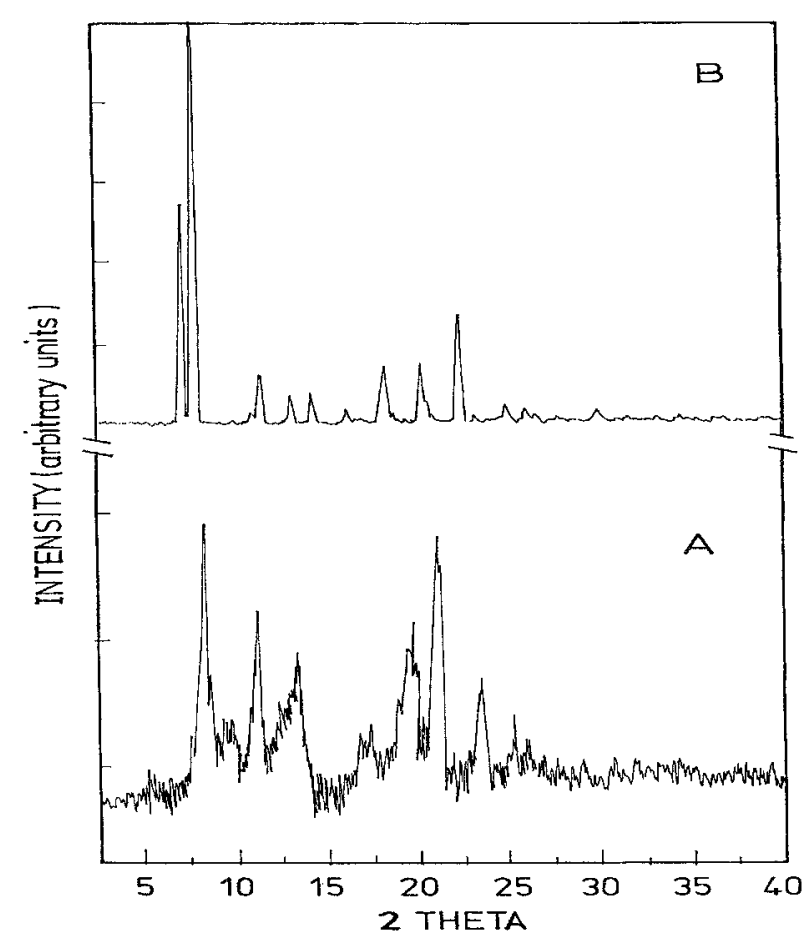

Figure 2. (A) Experimental and (B) simulated XRD patterns of PAPSP. 
Table I. The $d$ Spacing, Domain Length $(L), 2 \theta$, and Crystallographic Index $(h k l)$ Values of Monoclinic Indexation of PAPSP Obtained from Figure $2^{\mathrm{a}}$

\begin{tabular}{lcrrr}
\hline & $2 \theta^{\circ}$ & & & \\
(exp.) & (theo.) & $\begin{array}{c}d(\AA) \\
(\text { exp. })\end{array}$ & $\begin{array}{c}L(\AA) \\
\text { (exp.) }\end{array}$ & $\begin{array}{c}h k l \\
\text { (exp.) }\end{array}$ \\
\hline 7.42 & $7.90(\mathrm{~s})$ & 11.90 & 1133 & $(100)$ \\
8.20 & $8.60(\mathrm{vs})$ & 10.77 & 147 & $(010)$ \\
$11.18(\mathrm{~s})$ & $11.00(\mathrm{vw})$ & 7.91 & 103 & $(110)$ \\
13.31 & $13.85(\mathrm{w})$ & 6.64 & 68 & $(011)$ \\
17.22 & $17.15(\mathrm{w})$ & 5.15 & 84 & $(210)$ \\
19.59 & $18.90(\mathrm{~m})$ & 4.53 & 49 & $(021)$ \\
$20.95(\mathrm{~s})$ & $20.95(\mathrm{~m})$ & 4.24 & 201 & $(002)$ \\
23.33 & $23.20(\mathrm{~m})$ & 3.81 & - & $(102)$ \\
25.14 & $25.20(\mathrm{vw})$ & 3.54 & - & $(-311)$ \\
\hline
\end{tabular}

a The deviation in the intensity observed in the first peak appears to have a link with the large crystalline order $(L)$ shown by PAPSP. We believe that this is a characteristic of the poor crystallinity depicted by PAPSP and also of the extent to which the amorphous halo is accounted for in this $2 \theta$ range. $\mathrm{vs}=$ very strong; $\mathrm{s}=$ strong; $\mathrm{m}=$ medium; $\mathrm{w}=$ weak; $\mathrm{vw}$ $=$ very weak; exp. $=$ experimental; theo. $=$ theoretical.

\section{Theoretical Simulation of XRD Pattern}

An idealized model of the PAPSP oligomer of four repeat units was generated with a standard BIOSYM INSIGHT II package for calculating the theoretical XRD pattern. The model was minimized to obtain an optimized geometry that avoided steric repulsion between the neighboring rings. The presence of a tetrahedral geometry at the $\mathrm{C} 2$ atom (Fig. 3) makes the model a threedimensional object. Hence, this model has more than one parameter to be varied. On the basis of the symmetry of PAPSP, there are two possible crystalline conformers, namely the planar zigzag and helical structures. However, the planar zigzag is not tenable because of the presence of the peroxide bond. Because we assumed that the polymer would adopt the helical conformation, a possible space group in the monoclinic crystal system was $\mathrm{P} 2{ }_{1}$. A powder XRD pattern was simulated (Fig. 2) for the helical structure. Due to the poor crystallinity of PAPSP, there was fair agreement (Table I) between the experimentally observed XRD pattern and the simulated XRD, thus providing evidence that the structure of PAPSP is helical in nature. The highest peak was indexed as (010), which is a systematic absence in the space group $\mathrm{P} 2{ }_{1}$. The anomaly suggests that the crystal structure is pseudomonoclinic, with $\alpha$ and $\gamma$ being accidentally close to $90^{\circ}$. None of the space groups in the monoclinic symmetry conformed to the helical features depicted by the model except for the space group P2 . Furthermore, the deviations seen in the relative intensities between the experimental and simulated XRD patterns also may be due to these distortions in the features of the unit cell being enforced on the idealized model. The calculations made for theoretical powder patterns of other space groups in monoclinic symmetry, such as $\mathrm{Pm}, \mathrm{Pc}$, and $\mathrm{C} 2 / \mathrm{m}$, did not yield peaks at the experimentally observed $2 \theta$ values. Also, the first two peaks (i.e. $2 \theta=7.9$ and $8.6^{\circ}$ ) of the theoretical XRD pattern show a significant match between the observed and generated patterns that certainly proves the occurrence of a helical structure in PAPSP. To calculate the pitch of the helix, an idealized model with 20 repeat units of PAPSP was generated and optimized. This helix was right-handed with a pitch of $\sim 12.9 \AA$. However, the value of the cell dimension along the $b$ axis was $10.7 \AA$, which is clear proof for the occurrence of a pseudomonoclinic lattice that incorporates the helical dimensions. Additionally, some characteristic properties of the optimized structure were obtained. The minimized average bond lengths of $\mathrm{O}-\mathrm{O}, \underline{\mathrm{CPh}}{ }_{2}-\mathrm{OOCH}_{2}$,

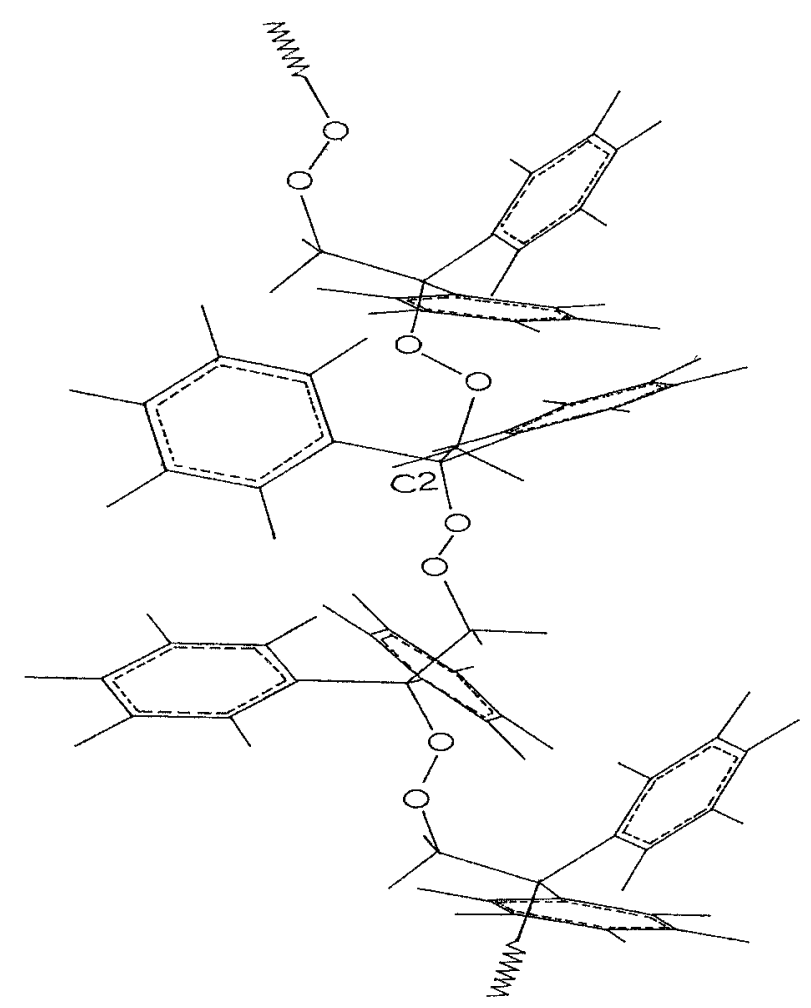

Figure 3. Chain segment of PAPSP showing a helical structure. 
and $\mathrm{O}-\mathrm{O}-\mathrm{CH}_{2}$ were $1.21,3.57$, and $2.21 \AA$, respectively. The average angles $\mathrm{O}-\mathrm{O}-\mathrm{CPh}_{2}$ and $\underline{\mathrm{O}}-\mathrm{O}-\mathrm{CH}_{2}$ were $117.24^{\circ}$ and $111.91^{\circ}$, respectively, and the average dihedral angle $(\phi)$ was $175.45^{\circ}$.

Of these properties, the dihedral angle $(\phi)$ is an important parameter of chemical interest. The chemical and physical properties of a peroxide partially depend on the $\phi$ value. ${ }^{17}$ Importantly, $\phi$ is sensitive to structure and molecular environment, and that can change the structure from an "open-book" to a "transplanar" conformation. A strong hydrogen-bonding interaction enlarges the $\phi$ value such that the molecule nearly adopts a transplanar conformation. ${ }^{17}$ Likewise, PAPSP exhibits a transplanar conformation at the $\mathrm{O}-\mathrm{O}$ bond because of its high $\phi$ value. The high $\phi$ value is attributable to the restriction imposed by the other part of the extended polymer chain on the open book structure, which eventually opens up to a transplanar conformation. The high $\phi$ value is, perhaps, a reason for the unusual chemical and physical properties exhibited by vinyl polyperoxides in contrast to simple monomeric organic peroxides.

\section{CONCLUSIONS}

By being crystalline and powdery in addition to having a higher thermal and photostability, PAPSP is perhaps the most eligible candidate for exploring novel application potentials. Unlike other polyperoxides that are generally stored in the cold, PAPSP can be stored safely at room temperature. Also, PAPSP is much less hazardous than simple organic peroxides. Because the helical structure is the most important secondary structure in biological macromolecules, any knowledge of the helical structure of synthetic polymers would be of considerable interest.

\section{REFERENCES AND NOTES}

1. Kishore, K.; Murthy, K. S. In Polymeric Materials Encyclopedia: Synthesis, Properties and Applications; Salamone, J. C., Ed.; CRC: New York, 1996; Vol. 11, p 8578.

2. Mogilevich, M. M. Russ Chem Rev 1979, 48, 199.

3. Kishore, K.; Mukundan, T. Nature 1986, 324, 130.

4. Murthy, K. S.; Kishore, K. Macromolecules 1996, 29,4859 .

5. Jayaseharan, J.; Kishore, K. J Am Chem Soc 1998, 120,825 .

6. Mayo, F. R. J Am Chem Soc 1958, 80, 2497.

7. Mayo, F. R.; Miller, A. A. J Am Chem Soc 1958, 80, 2480.

8. Caglioti, V.; Lenzi, M.; Mele, A. Nature 1964, 201, 610.

9. Staudinger, H. Ber 1925, 58, 1075.

10. Mayo, F. R.; Miller, A. A.; Russell, G. A. J Am Chem Soc 1958, 80, 2500.

11. Subramanian, K.; Kishore, K. J Polym Sci Part A: Polym Chem 1996, 34, 3361.

12. ${ }^{1} \mathrm{H}$ NMR ( $\left.\delta\right): 4.46$ (s, $\left.-\mathrm{CH}_{2}-\right) .{ }^{13} \mathrm{C} \mathrm{NMR}(\delta): 77.3$ $\left(\mathrm{s},-\mathrm{CH}_{2}-\right), 87.3(\mathrm{~s},>\mathrm{C}<)$. Fourier transform infrared: $1043 \mathrm{~cm}^{-1}(-\mathrm{O}-\mathrm{O}-$ absorption).

13. Werner, P. Z Kristallogr 1964, 120, 375.

14. Cullity, B. D. Elements of X-Ray Diffraction; Addison-Wesley: Reading, MA, 1956; p 99.

15. Pouget, J. P.; Jozefowics, M. E.; Epstein, A. J.; Tang, X.; MacDiarmid, A. G. Macromolecules 1991, 24, 779 .

16. Rietveld, H. M. Acta Crystallogr 1967, 22, 151.

17. Silbert, L. S. In Organic Peroxides; Swern, D., Ed.; Wiley: New York, 1971; Vol. II, p 637. 